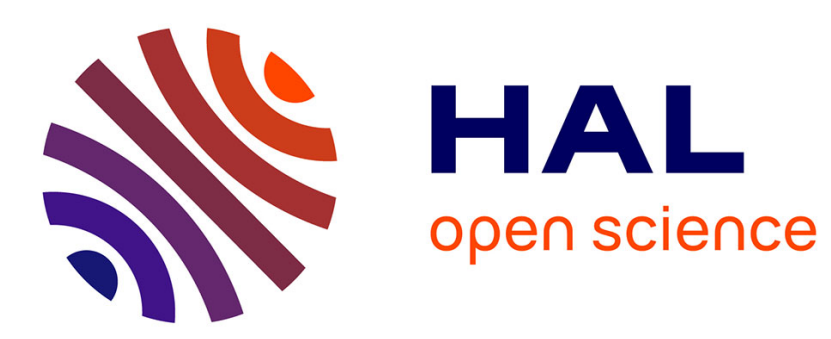

\title{
Epigenetic deregulation of genomic imprinting in humans: causal mechanisms and clinical implications
}

\author{
Michael Girardot, Robert Feil, David Llères
}

\section{To cite this version:}

Michael Girardot, Robert Feil, David Llères. Epigenetic deregulation of genomic imprinting in humans: causal mechanisms and clinical implications. Epigenomics, 2013, 5 (6), pp.715 - 728. 10.2217/epi.13.66 . hal-03027056

\section{HAL Id: hal-03027056 https://hal.science/hal-03027056}

Submitted on 26 Nov 2020

HAL is a multi-disciplinary open access archive for the deposit and dissemination of scientific research documents, whether they are published or not. The documents may come from teaching and research institutions in France or abroad, or from public or private research centers.
L'archive ouverte pluridisciplinaire $\mathbf{H A L}$, est destinée au dépôt et à la diffusion de documents scientifiques de niveau recherche, publiés ou non, émanant des établissements d'enseignement et de recherche français ou étrangers, des laboratoires publics ou privés. 


\section{Epigenetic deregulation of genomic imprinting in humans: causal mechanisms and clinical} implications

Mammalian genes controlled by genomic imprinting play important roles in development and diverse postnatal processes. A growing number of congenital disorders have been linked to genomic imprinting. Each of these is caused by perturbed gene expression at one principal imprinted domain. Some imprinting disorders, including the Prader-Willi and Angelman syndromes, are caused almost exclusively by genetic mutations. In several others, including the Beckwith-Wiedemann and Silver-Russell growth syndromes, and transient neonatal diabetes mellitus, imprinted expression is perturbed mostly by epigenetic alterations at 'imprinting control regions' and at other specific regulatory sequences. In a minority of these patients, DNA methylation is altered at multiple imprinted loci, suggesting that common trans-acting factors are affected. Here, we review the epimutations involved in congenital imprinting disorders and the associated clinical features. Trans-acting factors known to be causally involved are discussed and other trans-acting factors that are potentially implicated are also presented.

\section{KEYWORDS: Beckwith-Wiedemann syndrome DNA methylation epigenetics genomic imprinting pseudohypoparathyroidism Silver-Russell syndrome trans-acting factors transient neonatal diabetes mellitus ZFP57}

Almost 30 years ago, experiments in mice formally established that the maternal and paternal genomes are both required for embryonic and fetal development. Mouse embryos engineered to contain only maternal (gynogenetic/parthenogenetic embryos) or only paternal (androgenetic embryos), genomes failed to develop to term $[1,2]$, demonstrating the functional nonequivalence of the parental genomes. In addition, in other placental mammals, including humans, it was found that parthenogenetic (with two maternal genomes) and androgenetic (with two paternal genomes) conceptuses are developmentally aberrant and die in utero. The nonequivalence of the parental genomes in eutherians is due to genomic imprinting, an epigenetic phenomenon that gives rise to parent-of-origin-specific, monoallelic expression of genes during development [3]. So far, genomic imprinting has been observed in eutherians and marsupials, but not in monotremes, and approximately 120 imprinted genes have been discovered in humans, mice and other placental mammals [201]. These unusual genes are organized in evolutionarily conserved gene clusters that can comprise up to several megabases of DNA. The monoallelic expression of imprinted genes is mediated by so-called imprinting control regions' (ICRs). These essential cis-acting sequences of several kilobases in size are $\mathrm{CpG}$ rich and marked by cytosine methylation on one of the two parental alleles only. The monoallelic
DNA methylation imprints at ICRs are acquired during male or female gametogenesis. After fertilization, they are maintained somatically throughout development and postnatal life, which confers an epigenetic 'memory' to the ICR [3]. During embryonic development, the differentially methylated ICRs produce parental allele-specific expression at adjacent genes through diverse epigenetic mechanisms, which are different between imprinted domains [4]. Because of their allelic DNA methylation, most ICRs are functionally active on one of the two parental chromosomes only, which explains their parental allele-specific effects on gene expression [3,5].

Genetic alterations affecting imprinted gene expression include translocations, deletions, duplications, inversions and uniparental disomy (UPD), and have been shown to be causally involved in different imprinting disorders. Furthermore, epigenetic alterations were found to be causally involved in imprinting-related diseases. These so-called epimutations involve gains or losses of DNA methylation at ICRs and at other differentially methylated regions (DMRs), and affect the regulation of one or multiple imprinted domains. Although in most cases, such epimutations are thought to arise early in development and, in exceptional cases, are due to perturbed imprint acquisition in germ cells; their precise causes remain poorly understood [6]. Nevertheless, the aberrant DNA methylation

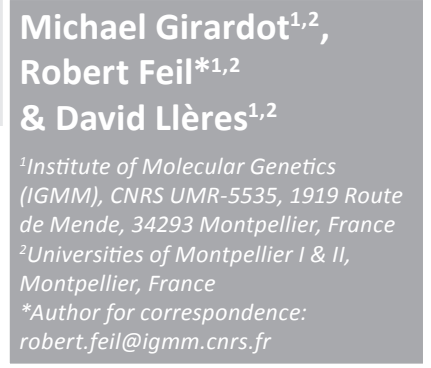

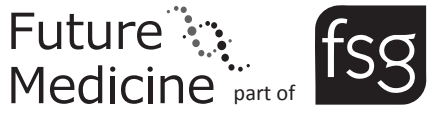


profiles at ICRs often result from perturbation of methylation maintenance mechanisms. Several studies in animals and humans have indicated that this intrinsic maintenance process can be readily influenced by environmental cues [5]. For instance, superovulation, embryo culture and other in vitro procedures used in the fertility clinic could be responsible for the reported increased occurrence of imprinting disorders in babies conceived by assisted reproduction [7].

DNA methylation changes at ICRs may also be the consequence of point mutations and microdeletions at imprinted gene loci [8], or may result from genetic mutations at genes elsewhere in the genome that code for essential trans-acting factors [9]. The latter possibility, that trans-acting factors are causally involved, has arisen from different reports of patients who presented with DNA hypomethylation at multiple ICRs (see 'Multilocus DNA methylation defects in imprinting disorders' section). During recent years, genetic studies in the mouse have pinpointed trans-acting proteins that are involved in the establishment and/or somatic maintenance of parental imprints at imprinted gene clusters. Recently, clinical studies in humans also revealed several genes that encode trans-acting factors involved in imprinting. Here, we review recent advances in our understanding of epigenetic perturbation of genomic imprinting in human congenital disorders and discuss the clinical implications. Genomic imprinting is also frequently perturbed in cancer; this related theme is covered in detail elsewhere [10] and is not part of the current review.

\section{Human disorders caused by epigenetic alterations at imprinted loci}

Epimutations at imprinted gene loci can be classified as primary or secondary alterations [11]. Primary epimutations concern direct modification of the methylation state of regulatory DNA sequences, affecting imprinted expression without changes in the DNA sequence. Secondary epimutations also involve aberrant DNA methylation and chromatin modifications, but these epigenetic changes are caused by adjacent genetic mutations at the imprinted locus itself, or by genetic mutations elsewhere in the genome controlling the epigenetic regulation of the imprinted loci in trans. In most cases, it is unclear whether an apparent primary epimutation is truly primary, since it is not known whether trans-acting factors are affected in the patient. Another complication in the interpretation of clinical studies is that DNA methylation levels can often only be determined in peripheral blood, and not in the actual tissues and organs affected by the disease. In many cases, therefore, it has been difficult to directly link ICR DNA methylation levels to the observed severity of the clinical phenotype in patients [12].

Primary epimutations at imprinted gene clusters involve the ICR, which shows either reduced or increased DNA methylation ('hypomethylation' and 'hypermethylation', respectively). In several imprinting-related disorders, this seems to be the most frequent causal mechanism [6]. Wellstudied examples are provided by two imprinted domains at the telomeric region of the short arm of chromosome 11 (11p15.5). These two growthrelated gene clusters are involved in Silver-Russell syndrome (SRS), characterized by intrauterine growth restriction (IUGR), and in BeckwithWiedemann syndrome (BWS), characterized by fetal overgrowth and high birth weight and height. A third imprinting disease, which is also often caused by primary epimutations, is transient neonatal diabetes mellitus (TNDM), a neonatal type of diabetes genetically linked to chromosome 6q24. Below, we review these rare congenital disorders caused most frequently by epigenetic mutations, and also briefly present several other imprinting-related diseases in which epimutations are less frequently involved.

\section{- Silver-Russell syndrome}

SRS (OMIM180860) is a rare (incidence one in $100,000[13])$ and mostly sporadic disorder characterized by IUGR, postnatal growth retardation, facial dysmorphism, feeding difficulties and body asymmetry [14]. It is a genetically heterogeneous disorder in which, in rare cases, maternal UPD of chromosome 7 and sometimes other chromosomal alterations are detected [15,16]. Recent epigenetic studies have linked this syndrome to one of the two imprinted gene clusters on human chromosome 11p15.5, the IGF2/H19 domain [17]. This growth-related locus comprises the paternally expressed IGF2 and INS (imprinted in the yolk sac only) genes and a growth-regulating ncRNA, called H19, expressed from the maternal chromosome. The domain is controlled by an intergenic ICR located several kilobases upstream of H19, called the H19-ICR. In 2005, for the first time, Gicquel et al. reported epimutations at the H19-ICR in SRS patients, namely, hypomethylation on the paternal allele, which induced a loss of IGF2 expression [17]. These results were confirmed by several subsequent studies $[18,19]$, indicating that more than $50 \%$ of SRS cases are caused by hypomethylation at the ICR of the 
IGF2/H19 locus, which provides a molecular explanation for this severe growth restriction syndrome [20]. Interestingly, complete [18] or partial $[17,18]$ hypomethylation was detected at the H19-ICR in SRS patients. This finding suggested that the loss of methylation occured early in development or could sometimes be caused by a constitutive genetic mutation(s). Whether other imprinted genes also contribute to SRS remains unclear. However, the maternal UPD of chromosome-7 observed in a minority of the patients suggests that a growth-regulating imprinted gene on this chromosome could also be involved. One candidate on this chromosome is the maternally expressed GRB10 gene [21]. A recent study reported an aberrant gain of DNA methylation at this gene and at several other imprinted genes in SRS patients [22]. Further studies are required to conclude whether or not GRB10 is genetically involved in SRS.

\section{Beckwith-Wiedemann syndrome} Contrary to SRS, BWS (OMIM 130650) is characterized by fetal and postnatal overgrowth and also presents an increased risk of childhood cancers (10\% of all patients). A minority of children with BWS develop Wilms' tumor (kidney cancer) and some present with adrenocortical carcinoma. Other clinical features of this rare syndrome (incidence one in 15,000 [23]) include macroglossia, midline abdominal wall defects, earlobe creases or ear pits and neonatal hypoglycemia [21,24,25]. BWS maps to chromosome 11p15. Besides the distal IGF2/H19 imprinted domain, this chromosomal region also comprises the large $(1 \mathrm{Mb})$ growth-regulating KCNQ1 imprinting cluster. The key gene in this second, proximally located cluster is CDKNIC (previously called p57KIP2), a negative regulator of the cell cycle. A frequently observed loss of CDKN1C expression in cancer suggests that this is also a tumor suppressor gene [10]. Approximately $5 \%$ of sporadic BWS cases and approximately $40 \%$ of cases with a positive family history present with point mutations in $C D K N 1 C$ [26,27]. Epimutations are responsible for approximately $60-70 \%$ of BWS cases. In approximately $10 \%$ of the patients, there is hypermethylation at the H19-ICR, which leads to biallelic (and therefore increased) IGF2 expression responsible for the observed overgrowth phenotype [20]. Strikingly, this is the exact opposite epigenetic change to that observed in SRS, in which this ICR loses its DNA methylation during early development (see 'Silver-Russell syndrome' section above). The most commonly observed epimutation in BWS, however, affects imprinting along the KCNQ1 imprinted domain, leading to a strong reduction in $C D K N 1 C$ expression. Particularly, at the domain's ICR (called the KvDMR1) there is loss of DNA methylation early in development. The lack of DNA methylation on both of the alleles of this ICR induces gene silencing along the domain on both the parental chromosomes, including that of $C D K N 1 C$. The resulting loss of CDKN1C expression is thought to be the main determinant in these cases of BWS, since deleterious point mutations in $C D K N 1 C$, on their own, also induce the overgrowth syndrome, in less than $10 \%$ of the patients [27].

A striking observation has been that cohorts of BWS babies present an increased frequency of monozygotic twinning. The affected twins are female and are discordant, with only one of the two girls showing the clinical features of the overgrowth syndrome [28]. Methylation studies have shown that there is often loss of methylation at the ICR of the KCNQ1 domain, with the epimutation occurring in the affected twin girl. Methylation changes in the peripheral blood, however, are often observed in both twins. In some monozygotic female twins, hypomethylation was also detected at the H19-ICR and the ICR of the DLK1-DIO3 imprinted domain [28]. Significantly, in SRS, discordant monozygotic female twins have been reported that have hypomethylation at the H19-ICR, again, affecting one of the two girls only [29]. One interpretation for this intriguing phenomenon is that the methylation losses somehow trigger the twinning process [28,29]. Alternatively, female preimplantation embryos are more prone to loss of DNA methylation than early male embryos, and this effect is exacerbated by the increased number of cell divisions due the monozygotic twinning process.

\section{Transient neonatal diabetes mellitus} TNDM (OMIM 601410) is a growth deficiency syndrome characterized by IUGR and persistent hyperglycemia during the first 6 weeks of life owing to a lack of insulin. This extremely rare syndrome (incidence <one in 100,000 [30]) has been linked to paternal UPD of chromosome 6 and to large duplications of region 6q24 on the paternal chromosome. In approximately $50 \%$ of cases, however, the disease seems to be not genetic in origin and is associated with defective DNA methylation at the imprinted transcription factor gene ZAC/PLAG1, localized on chromosome 6q24. Particularly, these TNDM patients with a normal karyotype show hypomethylation at exon 1 of $Z A C$ on the maternal chromosome $[31,32]$. This putative ICR region is thought 
to regulate the imprinted expression of $Z A C$ and of another, close-by imprinted gene called HYMA1 [33]. The resulting loss of imprinting (biallelic expression) of $Z A C$, particularly in the pancreas and/or pituitary, is thought to induce TNDM [34,35]. Specifically, the ZAC transcription factor is involved in cell-cycle control and insulin secretion in the pancreas. Mouse studies have shown that it regulates the expression of several other imprinted genes, including $\operatorname{Ig} f 2$ and $C d k n 1 c$, and influences the allelic silencing activity of the ICR of the Kcnq1 imprinted domain [36]. It is possibly through the effects of ZAC on other imprinted loci that TNDM shows clinical overlap with BWS (see also 'Multilocus DNA methylation defects in imprinting disorders' section).

\section{Pseudohypoparathyroidism}

Pseudohypoparathyroidism (PHP; OMIM 103580) represents a heterogeneous group of disorders whose common feature is end-organ resistance to parathyroid hormone leading to hypocalcemia, hyperphosphatemia and obesity [37]. Patients have a short stature, a rounded face and often mild mental retardation, and other characteristic features jointly termed Albright's hereditary osteodystrophy. These syndromes are due to genetic or epigenetic mutations affecting the GNAS locus on chromosome 20q13.11, which encodes the stimulatory G-protein subunit Gs $\alpha$, necessary for hormone signal transduction. GNAS transcription is predominantly biallelic with selective imprinting and alternative promoter usage in different tissues. PHPla arises upon maternal inheritance of molecular defects at GNAS. Epimutations - particularly loss of allelic DNA methylation at exon $1 \mathrm{~A}$ - result in PHP1b. PHP1b patients have renal parathyroid hormone resistance, decreased cAMP response to parathyroid hormone infusion and normal erythrocyte Gs activity, resulting in loss of the maternal allele expression in renal tissue, but without other features of Albright's hereditary osteodystrophy, presumably because activity is maintained in tissues where Gs $\alpha$ is expressed biallelically. Approximately $15-20 \%$ of PHP1b patients display a specific loss of methylation at the GNASDMRs, whereas most PHP1b patients (80-85\%) display a broad loss of imprinting at the GNAS domain $[38,39]$.

\section{- Prader-Willi syndome \& Angelman syndrome}

The Prader-Willi (PWS; OMIN 176270) [40] and Angelman (AS; OMIN 105830) [41,42] syndromes are rare neurodevelopmental genetic syndromes (incidence one in 10,000) and were the first reported imprinting diseases. They both map to human chromosome 15q11-13, a large imprinted domain comprising many genes that are expressed from the paternal chromosome in the brain, and one gene (UBE3A) expressed from the maternal chromosome. Whereas PWS is most often caused by maternal UPD of chromosome 15 , AS is caused most frequently by maternal deletion of chromosome 15q11-q13. The clinical phenotype of PWS is highly complex and caused by loss of expression of multiple paternally expressed genes on the domain. By contrast, AS is caused by loss of expression of the single maternally expressed gene $U B E 3 A$, which maps to $15 \mathrm{q} 11-13$. Since PWS and AS are caused almost exclusively by genetic mutations affecting the imprinted domain itself $[6,7]$, it has not been systematically explored in humans whether trans-acting factors could also be affected. Mouse studies, however, have identified several proteins involved in the embryonic maintenance of DNA methylation imprint at the domain's ICR (see 'Trans-acting factors involved in imprinting-related diseases' section).

\section{- Uniparental disomy 14 syndromes}

Maternal UPD of chromosome 14 is an extremely rare condition, which causes pre- and postnatal growth retardation, congenital hypotonia, joint laxity, motor delay and mild mental retardation. Paternal UPD of chromosome 14 (OMIM 608149) has a more severe phenotype, with polyhydramnios (excess of amniotic fluid), thoracic and abdominal wall defects, growth retardation and severe developmental delay. Cases of segmental UPD of chromosome 14 indicated that the distal portion of chromosome $14 \mathrm{q}$ is the critical region in these syndromes [43]. The $14 \mathrm{q} 32.2$ region contains a large cluster of imprinted genes that include the DLK1, RTL1 and $D I O 3$ genes expressed from the paternal chromosome, and the GTL2 (MEG3) and $M E G 8(R I A N)$, the antisense genes to RTL1 (RTL1as), and miRNA and snoRNA gene clusters expressed from the maternal chromosome [44]. This imprinted domain is under the control of an ICR called the intergenic germline DMR [45], which is methylated on the paternally inherited chromosome. Gene-targeting studies in mice suggest that excessive Rtll expression could explain the phenotype observed in patients with paternal UPD of chromosome 14, while decreased $D l k 1$ and Rtl1 gene expression could account for maternal UPD of chromosome 14 [43]. 
Several recent reports described deletions and epimutations affecting the imprinted DLK1$\mathrm{DIO} 3$ region at chromosome $14 \mathrm{q} 32.2 \mathrm{in}$ individuals with a phenotype similar to that of maternal UPD of chromosome 14 (but who did not present evidence for the occurrence of UPD) [46-48]. In these studies, loss of methylation was detected at the ICR (the intergenic germline-DMR) and also at the DMR comprising the $M E G 3$ promoter, indicating the occurrence of an epimutation (hypomethylation) affecting both of these paternally methylated DMRs [46-48].

\section{Multilocus DNA methylation defects in imprinting disorders}

In 2005, for the first time, Arima and his colleagues showed that the imprinted genes $Z A C$, KCNQ1OT1 (LIT1) and CDKN1C are likely part of a common imprinted gene network that plays a role in BWS [49]. They found that $Z A C$ bound to and activated the expression of another imprinted gene, KCNQ1OT1. The KCNQ1OT1 promoter corresponds to the ICR of the imprinted $K C N Q 1$ domain, and expression of this ncRNA negatively regulates the $C D K N 1 C$ gene in cis. The authors discovered that some patients with TNDM (two out of 17) had defects in DNA methylation at the KCNQ1OT1 promoter/ICR region, further supporting the proposed link between these two imprinted genes. Following this initial report, a breakthrough study on TNDM reported that besides loss of DNA methylation at $Z A C$, hypomethylation also occurred at several other imprinted gene loci, including at GRB10 (7p12), PEG1 (7q32), KvDMR1 (11p15; KCNQ1 domain) and PEG3 (19q13) [50]. The imprinted genes $S N R P N(15 \mathrm{q} 11-\mathrm{q} 13)$ and $H 19$ (11p15) remained unaltered however. In these patients, epigenetic mosaicism was inferred by the extent of the methylation defects and the gene loci and tissues that were affected. Interestingly, TNDM patients with multilocus methylation changes presented a higher weight at birth and a broader clinical spectrum in comparison to 'classical' TNDM patients. This could be explained by a deregulation of gene expression occurring at additional imprinted genes. Since there was loss of maternal allele-specific DNA methylation at all loci involved, it was hypothesized that the multilocus cases of TNDM might represent a disease syndrome of maternal hypomethylation. It is postulated that, in these patients, there is not only hypomethylation at ZAC1/PLAGL1, but also different degrees of hypomethylation at other ICRs and DMRs as well. The combined changes would make it difficult to readily link
DNA methylation levels at individual loci to a defined clinical etiology [50].

Recent studies on imprinting-related syndromes provide further evidence for multilocus methylation deregulation. One recent study carefully assayed allele-specific DNA methylation levels at 27 DMRs in a total of 65 patients with imprinting-related diseases [51]. The authors thus identified multilocus hypomethylation in numerous BWS, TNDM and PHP1b patients, and also in one individual with SRS. Other studies on SRS [52-54] revealed that 7\% of the patients with $\mathrm{H19}$ ICR hypomethylation also displayed hypomethylation at additional imprinted loci, and these changes frequently included the developmentally important DLK1-DIO3 imprinted locus [52]. Other recent work indicates that BWS patients show multilocus loss of DNA methylation in approximately $25 \%$ of the cases [55], and the most affected loci, besides KvDMR1 (the ICR of the KCNQ1 domain), are GNAS, IGF2R and PEG1 [56,57]. Similarly, a recent study reported that in some PHP1b patients, the loss of methylation at the GNASlocus had occurred concomitantly with lesser methylation changes at other imprinted loci [58]. Interestingly, in the latter study, both gains and losses of DNA methylation were reported. A recent publication, however, concluded that multilocus hypomethylation is an uncommon event in PHP patients [59]. Combined, novel data reveal the existence of a broad range of epimutations in several different imprinting-related disorders, which show partial overlap in their clinical phenotypes [51]. Based on these novel findings, by many different groups, hypothesis of 'epidominance' was put forward, in which the clinical phenotype is caused mostly by the imprinted locus at which the DNA methylation is most severely affected [58]. The new studies also emphasize that molecular testing for the above-described syndromes should not be restricted to the canonical causal locus [12].

Research has started to address whether genetic mutations in genes coding for transacting factors could explain the concomitant occurrence of methylation changes at multiple loci in some of the patients. So far, however, only few trans-acting factors have been causally linked to imprinting-related diseases. One of these is the zinc finger (ZF) protein, ZFP57, which is discussed below.

\section{Trans-acting factors involved in imprinting-related diseases}

As described previously, the involvement of trans-acting factors was first suspected when 
BWS and TNDM patients were found to present loss of DNA methylation at multiple loci other than the canonical loci affected in these diseases [9,56]. Below, we discuss the still limited number of trans-acting factors known to be perturbed in imprinting-related diseases. We also present trans-acting factors that emerged from recent animal studies whose involvement in human diseases remains to be explored. Trans-acting factors involved in imprinting maintenance are listed in Table 1. Their potential 'mode of action' in imprinting-related diseases is depicted in Figure 1.

\section{ZFP57 \& KAP1}

ZFP57 is a nuclear protein, which protects ICRs and other DMRs against loss of methylation in the embryo. Its key role initially emerged from studies on TNDM pedigrees, in which loss of DNA methylation at multiple DMRs was found to be linked to homozygous mutations in the ZFP57 gene [9,56]. Subsequently, additional loss-of-function mutations were identified at the human ZFP57 gene. A recently discovered 1-bp deletion resulting in premature translation termination and a truncated protein, were associated with hypomethylation at multiple maternal-methylated ICRs in TNDM patients [9,51]. Gene-targeting experiments in mice indicated that this KRAB-domain zinc-finger protein (ZFP) is required for the early embryonic maintenance of DNA methylation imprints [60], and binds to a methylated hexanucleotide motif (TGCCGC) that is present in most ICRs [61]. Indeed, similarly to the first and second ZFs, the third and fourth ZFs of human ZFP57 recognize the TGC ${ }^{\text {met }} \mathrm{CGC}$ motif, and two mutations (R248H and $\mathrm{H} 277 \mathrm{~N}$ ) associated with TNDM were shown to affect binding to this methylated DNA sequence [62]. This critical transcription factor is expressed in pluripotent cells and maintains the somatic methylation of imprinted domains through the recruitment of KAP1 (also called TRIM28 or TIF1 $\beta$ ), which recruits repressive chromatin modifiers, including ESET (also called SETDB1), a lysine

\begin{tabular}{|c|c|c|c|c|c|c|}
\hline Species & $\begin{array}{l}\text { Trans-acting } \\
\text { factor }\end{array}$ & Cofactor(s) & Function & $\begin{array}{l}\text { Phenotype due to } \\
\text { mutation/protein deficiency }\end{array}$ & $\begin{array}{l}\text { Human } \\
\text { disease }\end{array}$ & Ref. \\
\hline Human & ZFP57 & KAP1 & $\begin{array}{l}\text { Binds DNA at many } \\
\text { methylated ICRs }\end{array}$ & Hypomethylation of multiple ICRs & $\begin{array}{l}\text { TNDM } \\
\text { BWS }\end{array}$ & {$[9,51]$} \\
\hline Mouse & KAP1 & SETDB1 & $\begin{array}{l}\text { Scaffold protein for } \\
\text { heterochromatin factors }\end{array}$ & Hypomethylation of multiple ICRs & - & [64] \\
\hline Mouse & ZFP42 & - & $\begin{array}{l}\text { Pluripotency transcription } \\
\text { factor, similarity to YY1 }\end{array}$ & $\begin{array}{l}\text { Aberrant acquisition of DNA } \\
\text { methylation at Gnas and Peg3 }\end{array}$ & - & [5] \\
\hline \multirow[t]{2}{*}{ Human } & NLRP7 & C6orf221 & Unknown & Hypomethylation at multiple ICRs & $\mathrm{FBHM}$ & [74] \\
\hline & NLRP2 & - & Unknown & $\begin{array}{l}\text { Loss of methylation at KvDMR1 } \\
\text { ICR (KCNQ1 domain) }\end{array}$ & BWS & [67] \\
\hline Human & C6orf221 & NLRP7 & Unknown & $\begin{array}{l}\text { Hypomethylation at maternal } \\
\text { ICRs }\end{array}$ & $\mathrm{FBHM}$ & {$[76,77]$} \\
\hline Human & OCT4 & sox2 & $\begin{array}{l}\text { Pluripotency transcription } \\
\text { factor. Protection against } \\
\text { DNA methylation }\end{array}$ & $\begin{array}{l}\text { Mutation of OCT4/SOX2 sites at } \\
\text { specific ICRs leads to hyper- } \\
\text { methylation }\end{array}$ & BWS & {$[79,80]$} \\
\hline Mouse & CTCF & $\begin{array}{l}\text { Cohesin, } \\
\text { OCT4 }\end{array}$ & $\begin{array}{l}\text { Higher-order chromatin } \\
\text { organization }\end{array}$ & Gain of methylation at H19-ICR & - & [96] \\
\hline Mouse & DPPA3 & RanBP5 & $\begin{array}{l}\text { Protection against DNA } \\
\text { demethylation in mouse }\end{array}$ & $\begin{array}{l}\text { Hypomethylation at several } \\
\text { maternal and paternal ICRs in } \\
\text { mice }\end{array}$ & - & [99] \\
\hline Mouse & ARID4A \& ARID4B & SIN3A & $\begin{array}{l}\text { Maintenance of DNA } \\
\text { methylation and histone } \\
\text { methylation }\end{array}$ & $\begin{array}{l}\text { Loss of methylation at Snrpn ICR } \\
\text { (PWS domain) }\end{array}$ & - & [104] \\
\hline Mouse & MBD3, MTA2 & - & $\begin{array}{l}\text { Components of the NuRD } \\
\text { complex }\end{array}$ & $\begin{array}{l}\text { Hypomethylation at at H19-ICR } \\
\text { and Peg3 }\end{array}$ & - & {$[105,106]$} \\
\hline
\end{tabular}



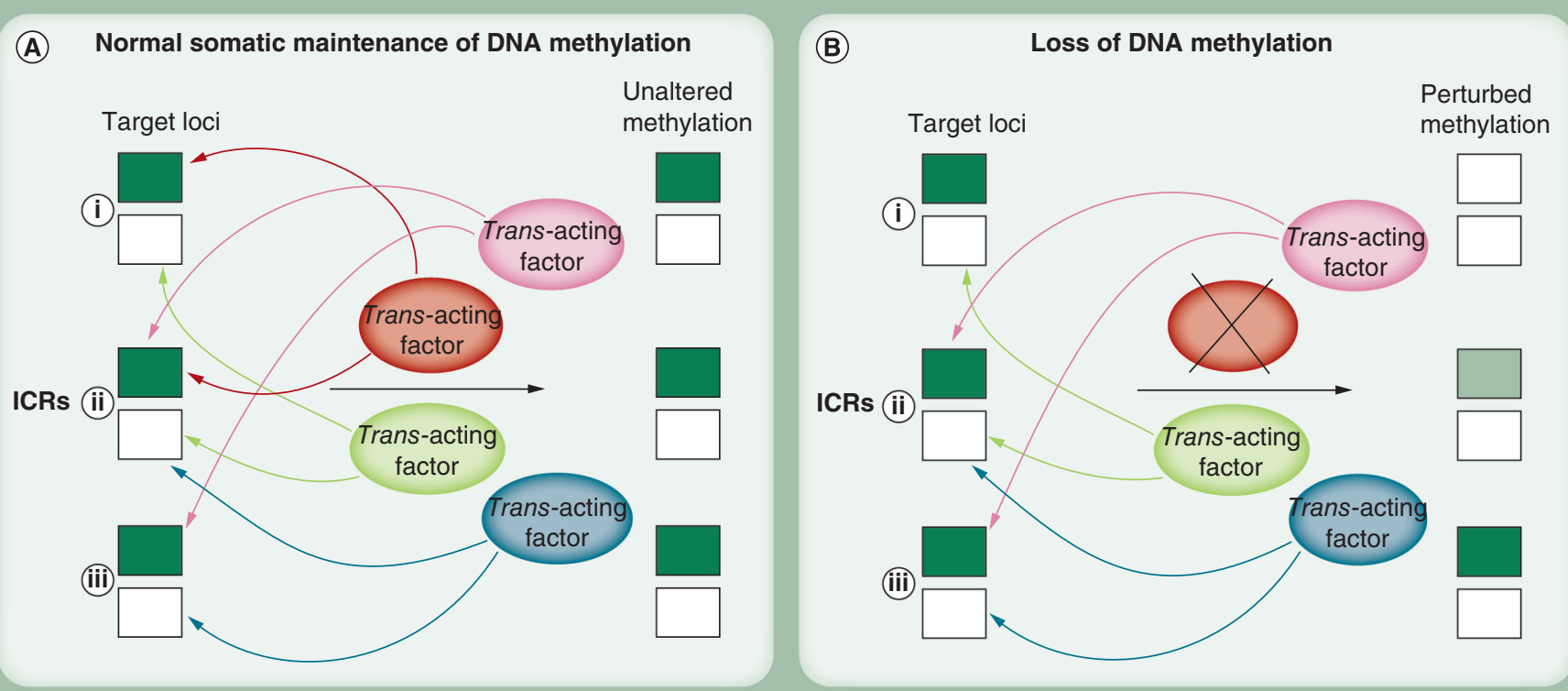

(C)

Gain of aberrant DNA methylation
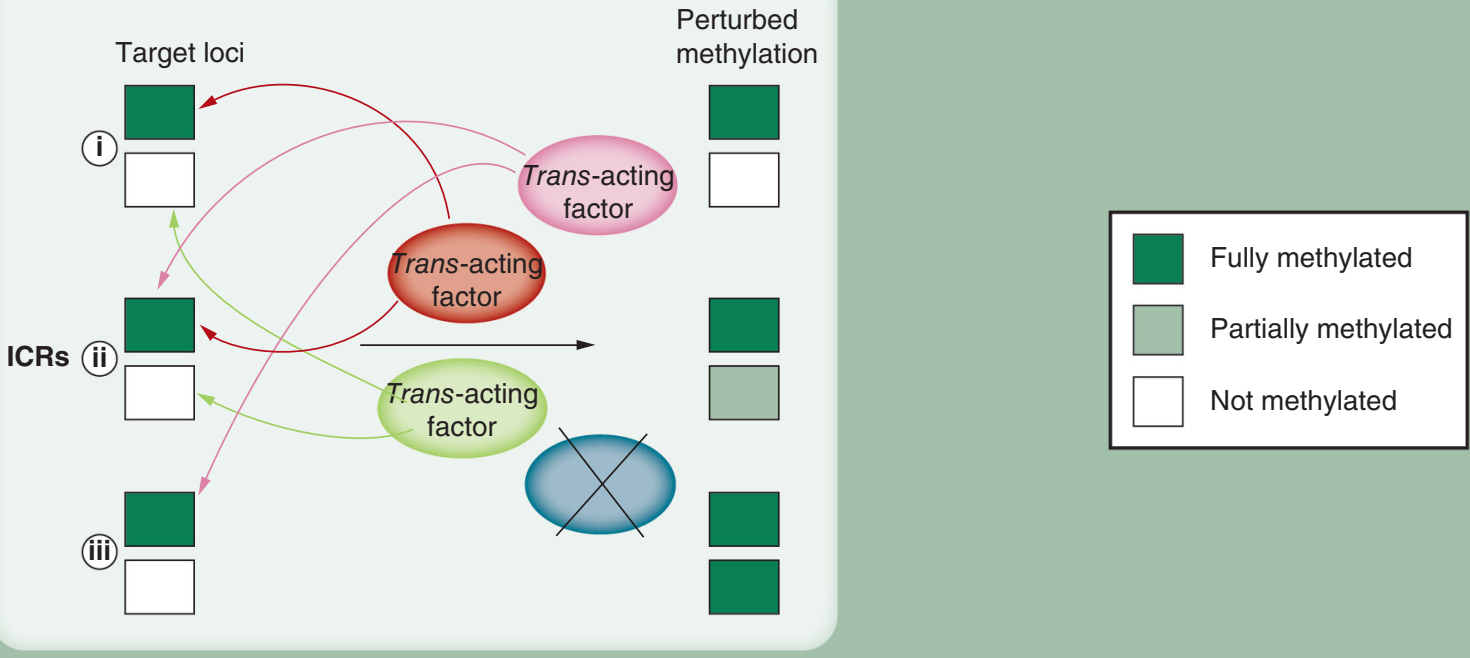

Figure 1. Involvement of trans-acting maintenance factors in imprinting-related diseases. (A) Normal maintenance of differential DNA methylation at ICRs. Multiple proteins ensure that the DNA-methylated alleles (dark green boxes) of ICRs remain methylated during development. Many of these trans-factors act on subsets of ICRs. For simplicity, three ICRs are depicted, with two methylation maintenance factors (red and pink circles) that have overlapping targets (left part of the figure). Conversely, the unmethylated alleles of ICRs need to remain unmethylated, which involves other trans-acting factors that protect against acquisition of de novo methylation. For simplicity, only two such protective trans-factors are depicted (green circles), each with a different combination of targets. The right part of the figure indicates that the allelic DNA methylation is maintained faithfully at the three ICRs. (B) Aberrant loss of DNA methylation at ICRs in imprinting diseases due to mutations in, or altered expression of, trans-acting factors. In the example given, the functional loss of one of the trans factors (indicated with a cross) leads to a complete loss of methylation at one, and partial loss of methylation at another of its target ICRs (right part of the figure). (C) Gain of aberrant DNA methylation in disease due to mutations in, or altered expression of, a trans-acting factor. In the example given, the functional loss of a protective trans-acting factor (depicted with a cross) leads to hypermethylation at one ICR, and a smaller gain of methylation at another ICR (right part of the figure). ICR: Imprinting control region.

For color figure, please see www.futuremedicine.com/doi/full/10.2217/epi.13.66

methyltransferase that deposits $\mathrm{H} 3 \mathrm{~K} 9 \mathrm{me} 3$ onto chromatin [61]. The latter repressive mark is recognized by the UHRF1 (also known as NP95 or ICBP90), which facilitates recruitment of the maintenance DNMT1 following DNA replication [63]. Although the precise relationship between these different protein factors remains unclear, combined, these proteins ensure that DNA methylation imprints are efficiently maintained, particularly during the critical preimplantation stages of development. Concordantly, in KAP1-deficient early mouse embryos and in cancer cells, loss of DNA methylation occurs at multiple ICRs $[64,65]$. 


\section{ZFP42}

Whether related ZF proteins could also be involved in the perturbation of DNA methylation imprints in disease, is unknown. In the mouse, however, loss of ZFP42 expression was found to induce aberrant acquisition of DNA methylation at the Gnas and Peg3 imprinted domains [66].

\section{NLRP2 \& NLRP7}

NLRP2 and NLRP7 (also called NALP2 and NALP7) encode proteins that comprise a nucleotide-binding oligomerization domain, a leucine-rich repeat and a pyrin domain (NLRP), and belong to a family of cytoplasmic proteins of unknown function comprising 14 members. $N L R P 2$ is highly homologous to NLRP7, and both proteins are expressed in oocytes and preimplantation embryos. In rare cases of BWS that presented loss of DNA methylation at the KvDMR1 ICR (the KCNQ1 domain), it was found that this methylation defect was linked to a frame-shift mutation in exon 6 of NLRP2 [67], revealing, for the first time, a trans mechanism in BWS. A complete hydatidiform mole represents a highly aberrant developmental phenotype resulting from a general perturbation of genomic imprinting. In most cases of complete hydatidiform mole, the conceptus is entirely androgenetic, with two paternal genomes [68]. In a similar way as observed in mice $[1,2]$, the lack of a maternal genome mostly results in proliferation of the extra-embryonic tissues, while embryonic development fails [69]. A rare familial, biparental form of this extremely severe clinical phenotype, named familial biparental hydatidiform mole (FBHM), has been identified, in which affected women suffer from recurrent abnormal pregnancy with no embryonic development and cystic degeneration of placental villi [70]. In different cases of FBHM, there was a failure to establish maternal imprints in the oocyte [71-73]. The imprint acquisition failure at multiple maternal ICRs suggested that a transacting factor was genetically affected. In 2006, recessive mutations in NLRP7 were identified as one cause of FBHM and further studies demonstrated that NLRP7 is mutated in a majority of FBHM families [74]. It remains to be determined precisely how NLRP7 contributes to maternal imprint establishment during oogenesis.

\section{C6orf221}

Some FBHM families do not present NLRP7 mutations, showing that there is genetic heterogeneity in familial hydatidiform moles [72,75].
Mutations in C6orf221 - a member of a rapidly evolving gene family specific to eutherians - were identified as a second genetic cause of FBHM [76]. C6orf221 (also called ECAT1) is part of a group of four highly similar genes within a $100-\mathrm{kb}$ cluster on human chromosome 6 [77]. Members of this gene family display a mostly oocyte and/or early embryonic expression pattern. This highly specific expression pattern, and the fact that genetic mutations in C6orf221 and NLRP7 induce identical phenotypes (i.e., absence of maternal imprints), make it likely that NLRP7 and C6orf221 participate in an oocyte-specific protein complex involved in imprint establishment. Indeed, the C6orf221 and NLRP7 proteins were found to interact [76]. Further studies are required to elucidate the precise molecular mechanisms, and to which extent these factors could also be important in the embryonic maintenance of imprints.

\section{Involvement of pluripotency factors}

Dynamic, genome-wide changes occur in DNA methylation during the preimplantation stages of development. Concomitant to these global processes, developmental pluripotency is acquired in the inner cell mass of the blastocyst. Despite the major transitions in the embryonic cells that are becoming pluripotent, remarkably, the differential epigenetic status of ICRs persists during preimplantation development [4]. This has raised the question as to whether pluripotency factors could be involved in epigenetic maintenance processes at imprinted loci. Recent genetic studies indicate that this is indeed the case. Point mutations and small deletions at OCT4- (also called POU5F1) and SOX2-binding sites in BWS patients at the H19-ICR were found to correlate with acquisition of DNA methylation at this ICR-controlling IGF2 expression [78-80]. In the reported cases, the defects were transmitted through the female germline only, with no phenotypic consequences following paternal transmission of the genetic mutations [79,80]. This interesting finding strongly suggests that binding of pluripotency factors to this ICR, on the unmethylated maternal allele, is essential in keeping this parental allele unmethylated through preimplantation development.

A potential role of OCT4 and SOX2 binding was also discovered at the ICR linked to the neurodevelopmental AS. In mechanistic studies in the mouse, mutation of either OCT4- or SOX2-binding sites resulted in a significant maternal allele-specific gain of methylation at this control region in oocytes [81]. 
Recently, it was experimentally confirmed that SOX2-OCT4 motifs (octamer motifs and their flanking sequences) in the ICR determine the cell type, DNA region and allele specificity of DNA methylation $[82,83]$. Another pluripotency factor, which contributes to the maintenance of the differential DNA methylation, at least in the mouse, is ZFP42 (also called REX1) [66]. This protein is highly expressed in the early embryo and is similar to the broadly expressed YY1 transcription factor.

Even if the mechanism of action of pluripotency factors themselves remains unclear, it is interesting to note that OCT4 and SOX2 binding has also been reported at the X-chromosome inactivation center, and both transcription factors are required for appropriate $\mathrm{X}$-chromosome inactivation [84]. OCT4 interacts directly with chromatin structural CTCF-cohesin complexes and its depletion induces inactivation on both $\mathrm{X}$ chromosomes upon differentiation of embryonic stem cells [84]. Mechanistically, OCT4 antagonizes cohesin binding. It thus acts as a negative regulator of chromosome-loop formation mediated by cohesin association to CTCFbinding sites [85]. It should be interesting to explore whether, besides their apparent protective effects against de novo DNA methylation, similar structural mechanisms are mediated by OCT4 binding to specific ICRs.

\section{- Involvement of CTCF \& cohesins}

CTCF is to be a major DNA-binding protein, which is able to establish insulators [86]. Specifically, CTCF binding can block enhancer function when it occurs between enhancers and promoters [87,88]. One of the best illustrations of such an enhancer-insulating function is provided by the H19/IGF2 locus. The differentially methylated ICR of this imprinted locus contains a cluster of CTCF-binding sites, and imprinted $\mathrm{H19} /$ IGF2 expression is regulated by selective binding of CTCF to the unmethylated allele. CTCF binding on the maternal chromosome mediates an allele-specific higher-order chromatin configuration at the domain and thus constitutes an enhancer-blocking transcriptional insulator of the IGF2 gene [89,90]. By contrast, methylation of the paternal allele prevents CTCF binding and thus prevents the establishment of the insulator function, allowing IGF2 expression on this parental chromosome [89-92]. Several genomewide studies have shown frequent colocalization of CTCF and cohesin, and CTCF binding is strictly required to recruit cohesin to the chromatin [93]. Conversely, cohesin seems to play a critical role in maintaining CTCF-mediated chromatin conformation [94]. Mutation of all four CTCF-binding sites in the H19-ICR leads to aberrant gain of DNA methylation on the maternal allele during postimplantation development [95,96]. In BWS, small maternally inherited ICR deletions $(1.4-2 \mathrm{~kb})$ that remove one or more of the CTCF sites were reported to be associated with an aberrant gain of DNA methylation at the remaining target sites, leading to increased expression of $I G F 2$ [97,98].

\section{DPPA3}

Mouse studies have shown that maternal proteins contribute to the protection against DNA demethylation following fertilization. DPPA3 (also called PGC7 or STELLA) is highly expressed during oogenesis and persists in the preimplantation embryo. Gene targeting experiments in the mouse have shown that after fertilization, up to the two-cell stage, Dppa3 plays a crucial role in protecting the maternal genome against DNA demethylation. This action includes the maternally methylated ICRs at the Peg1 (Mest), Peg3 and Peg10 loci. Additionally, on the paternal genome, DPPA3 protects the paternally methylated gene loci Dlk1-Dio3, H19-Igf2 and Rasgrf1 against active DNA demethylation in the early embryo [99,100]. Interestingly, DNA methylation of H19 and Rasgrf1 ICRs was not maintained in pronuclear five-stage embryos derived from DPPA3-null oocytes, whereas that of the Dlk1-Dio3 domain ICR was. These observations suggest that the mechanisms of epigenetic maintenance at the H19-Igf2 and Rasgrf1 domains could differ from that at the Dlk1-Dio3 domain. Possibly, some of the observed effects could also be linked to the reported role of DPPA3 in chromatin condensation during oogenesis [101], which might trigger additional histone modifications that protect against DNA demethylation. Interestingly, chromatin immunoprecipitation studies showed that the repressive $\mathrm{H} 3 \mathrm{~K} 9 \mathrm{me} 2$ is present at the $\mathrm{H} 19$ and Rasgrf1 ICRs, but not at the ICR of the Dlk1-Dio3 domain [102]. DPPA3 appears to protect the maternal genome against the TET3 protein-mediated conversion of 5-methylcytosine to 5-hydroxymethylcytosine through its competing association with $\mathrm{H} 3 \mathrm{~K} 9 \mathrm{me} 2$-marked chromatin $[102,103]$. Combined, the studies are consistent with a model in which inhibition of TET3 activity, through the binding of DPPA3 to $\mathrm{H} 3 \mathrm{~K} 9 \mathrm{me} 2$, provides protection against DNA demethylation during the very early stages of development. 


\section{ARID4A \& ARID4B}

Arid $4 A$ and Arid $4 B$ are closely related members of the ARID gene family. They encode RBP1 and RBP1L1, respectively, which are both members of chromatin-remodeling complexes and could recruit the histone deacetylase-dependent SIN3A transcriptional corepressor. Targeted deletion of both of these genes in mice resulted in loss of DNA methylation and reduced $\mathrm{H} 3 \mathrm{~K} 9 \mathrm{me} 3$ and H4K20me3 on the maternal allele at the Snrpn ICR controlling the PWS imprinted domain [104]. The study suggests that these proteins are involved in the maintenance of imprinting; however, their function in humans remains to be explored.

Other chromatin-associated proteins Several other chromatin regulatory proteins are involved in the embryonic maintenance of allelic DNA methylation at individual ICRs in the mouse. These include two components of the NuRD complex: MBD3 and MTA2. Knockdown studies were performed in embryonic cells on both of these proteins and this was found to lead to a partial loss of the paternal DNA methylation at the H19-ICR, but not at several other ICRs $[105,106]$. These data suggest that recruitment of the NuRD chromatin remodeling complex contributes to the DNA methylation at the H19-ICR, and therefore constitutes a candidate for involvement in SRS.

\section{Conclusion \& future perspective}

Several novel insights have emerged into the genetic and epigenetic origins of imprinting disorders. Possibly the most relevant novel finding is that in some patients, altered DNA methylation affects not only the canonical ICR involved in the disease, but multiple other ICRs/DMRs are affected to different extents. A multilocus aberrant DNA methylation phenotype has now been reported in different diseases and strongly suggests that common trans-acting factors are genetically affected in some of the patients. One major challenge for the future will be to identify these factors. Besides the exceptional involvement of ZFP57, no other trans-acting maintenance factor has been reported to be mutated in the multilocus methylation phenotype. A recent study on the DNMT3-L protein (involved in imprint establishment) shows that this candidate protein is not involved in aberrant multilocus methylation [51], but few other systematic studies have been performed on other candidates so far.

The recent reports on H19-ICR microdeletions and point mutations in BWS raise the question of what percentage of patients presenting small genetic mutations, including single nucleotide changes, could be responsible for the observed epimutations (hypomethylation and hypermethylations) at specific ICRs? To address this issue, it should be relevant in future studies to not only assess DNA methylation levels at ICRs, but to also carefully study their DNA sequences.

The novel insights also present additional challenges for the future. For instance, are changes confined to specific ICRs, and which ones, or are other DMRs in the genome affected? New technologies are already being developed to systematically assess DNA methylation at multiple ICRs and DMRs [31] or to detect UPDs with a high sensitivity for the estimation of their size and lowrate mosaicism [51,107]. Ideally, such studies should encompass all of the regulatory gene regions of the genome, an approach which becomes feasible with the emergence of affordable high-throughput sequencing approaches [108]. Additionally, it should be interesting to explore repressive histone modifications, particularly those that are linked to the somatic maintenance of DNA methylation imprints [109]. A major handicap remains the limiting availability of suitable tissue material, particularly to address whether aberrant methylation patterns are tissue-specific or are detected broadly in different lineages.

The occurrence of ICR point mutations and microdeletions highlights the importance of 'hunting' for genetic defects in cis in case the methylation change is confined to the canonical ICR associated with the imprinting disorder. In case the methylation change affects multiple imprinted (and nonimprinted) gene loci, however, it should seem relevant to explore whether trans-acting factor genes are mutated or altered in their expression. In such genetic studies, it might not be sufficient to include only the known transacting factors of imprinting disorders. Novel candidates emerging from mouse studies could be included in a targeted screen as well. Particularly when a familial component is suspected, it should be worthwhile to use broad, high-throughput sequencing approaches for mutation detection (e.g., exome sequencing). Hopefully, in the years to come, it will become clearer how commonly trans-acting factors are perturbed in imprintingrelated diseases in comparison with other causal mechanisms. Despite the many technological challenges ahead, these are exciting novel avenues of research. The coming years will undoubtedly pinpoint new players involved in imprintingrelated diseases, and may shed further light on the extent to which these epigenetic diseases could be genetic in origin. 


\section{Financial \& competing interests disclosure}

This work was grant supported by the Agence Nationale de Recherche, the Institut National de Cancer, the Fondation pour la Recherche Médicale (to R Feil) and the University of Montpellier I (to M Girardot). The authors' laboratory is affiliated with the European NoE 'EpiGeneSys' and the LABEX 'EpiGenMed'. The authors have no other relevant affiliations or financial involvement with any organization or entity with a financial interest in or financial conflict with the subject matter or materials discussed in the manuscript apart from those disclosed.

No writing assistance was utilized in the production of this manuscript.

\section{Executive summary}

\section{Imprinting disorders}

- Many congenital disorders are caused by perturbed imprinted gene expression. Some of these complex diseases are caused almost exclusively by genetic mutations at specific imprinted domains

- Other imprinting disorders seem to be caused mostly by epigenetic alterations ('epimutations').

\section{Epimutations that produce imprinting disorders}

- DNA methylation defects at specific imprinted gene domains are causally involved in several imprinting disorders.

- In most cases, there is decreased or increased DNA methylation at imprinting control regions; the cis-regulatory elements that control imprinted gene expression.

- Although the reported DNA methylation changes at imprinting control regions are often not accompanied by gross genetic changes, there could nevertheless be a genetic origin of the epimutation(s) in many of the patients.

\section{Multilocus DNA methylation defects in some patients}

- Several recent studies show that in some patients with specific imprinting disorders, methylation defects occur in concert at multiple imprinted loci.

- This novel finding strongly suggests that common trans-acting factors are (genetically) affected in these patients.

\section{Trans-acting factors involved in imprinting disorders}

- Several recent studies have identified trans-acting factors that are mutated in some patients with human imprinting disorders.

- Other candidate trans-acting factors have emerged from recent animal studies.

\section{References}

Papers of special note have been highlighted as:

- of interest

"w of considerable interest

1 McGrath J, Solter D. Completion of mouse embryogenesis requires both the maternal and paternal genomes. Cell 37(1), 179-183 (1984).

- Surani MA, Barton SC, Norris ML. Development of reconstituted mouse eggs suggests imprinting of the genome during gametogenesis. Nature 308(5959), 548-550 (1984).

- 3 Ferguson-Smith AC. Genomic imprinting: the emergence of an epigenetic paradigm. Nat. Rev. Genet. 12(8), 565-575 (2011).

4 Kacem S, Feil R. Chromatin mechanisms in genomic imprinting. Mamm. Genome 20(9-10), 544-556 (2009).

5 Feil R, Fraga MF. Epigenetics and the environment: emerging patterns and implications. Nat. Rev. Genet. 13(2), 97-109 (2011).

-6 Hirasawa R, Feil R. Genomic imprinting and human disease. Essays Biochem. 48(1), 187-200 (2010).

7 Arnaud P, Feil R. Epigenetic deregulation of genomic imprinting in human disorders and following assisted reproduction. Birth Defects Res. C Embryo Today 75(2), 81-97 (2005).
De Crescenzo A, Sparago A, Cerrato F et al. Paternal deletion of the $11 \mathrm{p} 15.5$ centromericimprinting control region is associated with alteration of imprinted gene expression and recurrent severe intrauterine growth restriction. J. Med. Genet. 50(2), 99-103 (2013).

\. Mackay DJ, Callaway JL, Marks SM et al. Hypomethylation of multiple imprinted loci in individuals with transient neonatal diabete is associated with mutations in ZFP57. Nat. Genet. 40(8), 949-951 (2008).

-n This study on transient neonatal diabetes mellitus shows that mutations at the ZFP57 (transcription factor) gene affect the somatic maintenance of DNA methylation at multiple imprinting control regions.

$\downarrow_{10}$ Uribe-Lewis S, Woodfine K, Stojic L, Murrel A. Molecular mechanisms of genomic imprinting and clinical implications for cancer. Expert Rev. Mol. Med. 13, e2 (2011).

11 Horsthemke B. Epimutations in human disease. Curr. Top. Microbiol. Immunol. 310, 45-59 (2006).

12 Azzi S, Rossignol S, Le Bouc Y, Netchine I. Lessons from imprinted multilocus loss of methylation in human syndromes: a step toward understanding the mechanisms underlying these complex diseases. Epigenetics 5(5), 373-377 (2010).
3 Abu-Amero S, Monk D, Frost J, Preece M, Stanier P, Moore GE. The genetic aetiology of Silver-Russell syndrome. J. Med. Genet. 45(4), 193-199 (2008).

14 Christoforidis A, Maniadaki I, Stanhope R. Managing children with Russell-Silver syndrome: more than just growth hormone treatment? J. Pediatr. Endocrinol. Metab. 18(7), 651-652 (2005).

Eggermann T. Russell-Silver syndrome. Am. J. Med. Genet. C Semin. Med. Genet.154C(3), 355-364 (2010).

16 Bruce S, Hannula-Jouppi K, Puoskari M et al. Submicroscopic genomic alterations in Silver-Russell syndrome and Silver-Russell-like patients. J. Med. Genet. 47(12), 816-822 (2010).

Gicquel C, Rossignol S, Cabrol S et al. Epimutation of the telomeric imprinting center region on chromosome $11 \mathrm{p} 15$ in Silver-Russell syndrome. Nat. Genet. 37(9), 1003-1007 (2005).

18 Bliek J, Terhal P, Van Den Bogaard MJ et al. Hypomethylation of the $\mathrm{H} 19$ gene causes not only Silver-Russell syndrome (SRS) but also isolated asymmetry or an SRS-like phenotype. Am. J. Hum. Genet. 78(4), 604-614 (2006).

19 Schonherr N, Meyer E, Eggermann K, Ranke MB, Wollmann HA, Eggermann T. (Epi)mutations in 11p15 significantly 
contribute to Silver-Russell syndrome: but are they generally involved in growth retardation? Eur. J. Med. Genet. 49(5), 414-418 (2006).

20 Feil R. Epigenetic asymmetry in the zygote and mammalian development. Int. J. Dev. Biol. 53(2-3), 191-201 (2009).

21 Butler MG. Genomic imprinting disorders in humans: a mini-review. J. Assist. Reprod. Genet. 26(9-10), 477-486 (2009).

22 Dias RP, Bogdarina I, Cazier JB et al. Multiple segmental uniparental disomy associated with abnormal DNA methylation of imprinted loci in Silver-Russell syndrome. J. Clin. Endocrinol. Metab. 97(11), e2188-e2193 (2012).

23 Weksberg R, Shuman C, Beckwith JB. Beckwith-Wiedemann syndrome. Eur. J. Hum. Genet. 18(1), 8-14 (2010).

24 Thorburn MJ, Wright ES, Miller CG, Smith-Read EH. Exomphalos-macroglossiagigantism syndrome in Jamaican infants. Am. J. Dis. Child. 119(4), 316-321 (1970).

${ }_{25}$ Wiedemann HR. Frequency of Wiedemann-Beckwith syndrome in Germany; rate of hemihyperplasia and of tumors in affected children. Eur. J. Pediatr. 156(3), 251 (1997).

-26 O’Keefe D, Dao D, Zhao L et al. Coding mutations in $\mathrm{p} 57 \mathrm{KIP} 2$ are present in some cases of Beckwith-Wiedemann syndrome but are rare or absent in Wilms tumors. Am. J. Hum. Genet. 61(2), 295-303 (1997).

- 27 Lee MP, Debaun M, Randhawa G, Reichard BA, Elledge SJ, Feinberg AP. Low frequency of $\mathrm{p} 57 \mathrm{KIP} 2$ mutation in Beckwith-Wiedemann syndrome. Am. J. Hum. Genet. 61(2), 304-309 (1997).

28 Bliek J, Alders M, Maas SM et al. Lessons from BWS twins: complex maternal and paternal hypomethylation and a common source of haematopoietic stem cells. Eur. J. Hum. Genet. 17(12), 1625-1634 (2009).

29 Yamazawa K, Kagami M, Fukumi M et al. Monozygotic female twins discordant for Silver-Russell syndrome and hypomethylation of the H19-DMR. J. Hum. Genet. 53(10), 950-955 (2008).

30 Shield JP. Neonatal diabetes: new insights into aetiology and implications. Horm. Res. 53(Suppl. 1), 7-11 (2000).

31 Temple IK, Shield JP. Transient neonatal diabetes, a disorder of imprinting. J. Med. Genet. 39(12), 872-875 (2002).

- 32 Arima T, Drewell RA, Arney KL et al. A conserved imprinting control region at the HYMAI/ZAC domain is implicated in transient neonatal diabetes mellitus. Hum. Mol. Genet. 10(14), 1475-1483 (2001).

33 Varrault A, Bilanges B, Mackay DJ et al. Characterization of the methylation-sensitive promoter of the imprinted $Z A C$ gene supports its role in transient neonatal diabetes mellitus. J. Biol. Chem. 276(22), 18653-18656 (2001).

34 Kamiya M, Judson H, Okazaki Y et al. The cell cycle control gene $Z A C / P L A G L 1$ is imprinted - a strong candidate gene for transient neonatal diabetes. Hum. Mol. Genet. 9(3), 453-460 (2000).

-35 Piras G, El Kharroubi A, Kozlov S et al. Zac1 (Lot1), a potential tumor suppressor gene, and the gene for epsilon-sarcoglycan are maternally imprinted genes: identification by a subtractive screen of novel uniparental fibroblast lines. Mol. Cell. Biol. 20(9), 3308-3315 (2000).

- 36 Varrault A, Gueydan C, Delalbre A et al. Zac1 regulates an imprinted gene network critically involved in the control of embryonic growth. Dev. Cell 11(5), 711-722 (2006).

"I- This mouse study shows that the imprinted gene $Z a c 1$, which encodes a trans-acting transcription factor, controls the expression of multiple other imprinted genes. It provides evidence for an imprinted gene network.

- 37 Kelsey G. Imprinting on chromosome 20: tissue-specific imprinting and imprinting mutations in the GNAS locus. Am. J. Med. Genet. C Semin. Med. Genet. 154C(3), 377-386 (2010).

- 38 Bastepe M, Frohlich LF, Linglart A et al. Deletion of the NESP55 differentially methylated region causes loss of maternal GNAS imprints and pseudohypoparathyroidism type Ib. Nat. Genet. 37(1), 25-27 (2005).

-39 Chillambhi S, Turan S, Hwang DY, Chen HC, Juppner H, Bastepe M. Deletion of the noncoding GNAS antisense transcript causes pseudohypoparathyroidism type Ib and biparental defects of GNAS methylation in cis. J. Clin. Endocrinol. Metab. 95(8), 3993-4002 (2010).

40 Clarren SK, Smith DW. Prader-Willi syndrome. Variable severity and recurrence risk. Am. J. Dis. Child. 131(7), 798-800 (1977).

41 Clayton-Smith J. Clinical research on Angelman syndrome in the United Kingdom: observations on 82 affected individuals. Am. J. Med. Genet. 46(1), 12-15 (1993).

42 Petersen MB, Brondum-Nielsen K, Hansen LK, Wulff K. Clinical, cytogenetic, and molecular diagnosis of Angelman syndrome: estimated prevalence rate in a Danish county. Am. J. Med. Genet. 60(3), 261-262 (1995).

43 Kagami M, Sekita Y, Nishimura G et al. Deletions and epimutations affecting the human 14q32.2 imprinted region in individuals with paternal and maternal upd(14)-like phenotypes. Nat. Genet. 40(2), 237-242 (2008).
44 Girardot M, Cavaille J, Feil R. Small regulatory RNAs controlled by genomic imprinting and their contribution to human disease. Epigenetics 7(12), 1341-1348 (2012).

45 Lin SP, Youngson N, Takada S et al. Asymmetric regulation of imprinting on the maternal and paternal chromosomes at the Dlk1-Gtl2 imprinted cluster on mouse chromosome 12. Nat. Genet. 35(1), 97-102 (2003).

46 Buiting K, Kanber D, Martin-Subero JI et al. Clinical features of maternal uniparental disomy 14 in patients with an epimutation and a deletion of the imprinted DLK1/GTL2 gene cluster. Hum. Mutat. 29(9), 1141-1146 (2008).

47 Zechner U, Kohlschmidt N, Rittner G et al. Epimutation at human chromosome 14q32.2 in a boy with a UPD(14)mat-like clinical phenotype. Clin. Genet. 75(3), 251-258 (2009).

48 Hosoki K, Ogata T, Kagami M, Tanaka T, Saitoh S. Epimutation (hypomethylation) affecting the chromosome 14q32.2 imprinted region in a girl with UPD(14)mat-like phenotype. Eur. J. Hum. Genet. 16(8), 1019-1023 (2008).

49 Arima T, Kamikihara T, Hayashida T et al. ZAC, LIT1 (KCNQ1OT1) and $p 57$ KIP2 $(C D K N 1 C)$ are in an imprinted gene network that may play a role in Beckwith-Wiedemann syndrome. Nucleic Acids Res. 33(8), 2650-2660 (2005).

- Reports that several imprinted genes are part of a common gene expression network, which is perturbed in BeckwithWiedemann syndrome.

50 Mackay DJ, Hahnemann JM, Boonen SE et al. Epimutation of the TNDM locus and the Beckwith-Wiedemann syndrome centromeric locus in individuals with transient neonatal diabetes mellitus. Hum. Genet. 119(1-2), 179-184 (2006).

- 51 Court F, Martin-Trujillo A, Romanelli V et al. Genome-wide allelic methylation analysis reveals disease-specific susceptibility to multiple methylation defects in imprinting syndromes. Hum. Mutat. 34(4), 595-602 (2013).

-III Shows the occurrence of multilocus hypomethylation in different imprinting disorders.

52 Azzi S, Rossignol S, Steunou V et al. Multilocus methylation analysis in a large cohort of 11p15-related foetal growth disorders (Russell Silver and Beckwith Wiedemann syndromes) reveals simultaneous loss of methylation at paternal and maternal imprinted loci. Hum. Mol. Genet. 18(24), 4724-4733 (2009). 
- Explores the extent of multilocus DNA hypomethylation in cohorts of Beckwith-Wiedemann syndrome and Silver-Russell syndrome patients.

-53 Turner CL, Mackay DM, Callaway JL et al. Methylation analysis of 79 patients with growth restriction reveals novel patterns of methylation change at imprinted loci. Eur. J. Hum. Genet. 18(6), 648-655 (2010).

-54 Demars J, Gicquel C. Epigenetic and genetic disturbance of the imprinted $11 \mathrm{p} 15$ region in Beckwith-Wiedemann and Silver-Russell syndromes. Clin. Genet. 81(4), 350-361 (2012).

-55 Netchine I, Rossignol S, Azzi S, Brioude F, Le Bouc Y. Imprinted anomalies in fetal and childhood growth disorders: the model of Russell-Silver and Beckwith-Wiedemann syndromes. Endocr. Dev. 23, 60-70 (2012).

-56 Rossignol S, Steunou V, Chalas C et al. The epigenetic imprinting defect of patients with Beckwith-Wiedemann syndrome born after assisted reproductive technology is not restricted to the $11 \mathrm{p} 15$ region. J. Med. Genet. 43(12), 902-907 (2006).

- 57 Bliek J, Verde G, Callaway J et al. Hypomethylation at multiple maternally methylated imprinted regions including PLAGL1 and GNAS loci in Beckwith-Wiedemann syndrome. Eur. J. Hum. Genet. 17(5), 611-619 (2009).

-58 Maupetit-Mehouas S, Azzi S, Steunou V et al. Simultaneous hyper- and hypomethylation at imprinted loci in a subset of patients with GNAS epimutations underlies a complex and different mechanism of multilocus methylation defect in pseudohypoparathyroidism type 1b. Hum. Mutat. 34(8), 1172-1180 (2013).

-59 Perez-Nanclares G, Romanelli V, Mayo S et al. Detection of hypomethylation syndrome among patients with epigenetic alterations at the GNAS locus. J. Clin. Endocrinol. Metab. 97(6), e1060-e1067 (2012).

-60 Li X, Ito M, Zhou F et al. A maternal-zygotic effect gene, $Z f p 57$, maintains both maternal and paternal imprints. Dev. Cell 15(4), 547-557 (2008).

-61 Quenneville S, Verde G, Corsinotti A et al. In embryonic stem cells, ZFP57/KAP1 recognize a methylated hexanucleotide to affect chromatin and DNA methylation of imprinting control regions. Mol. Cell 44(3), 361-372 (2011).

- Explores the molecular mechanisms through which ZFP57 and KAP1 maintain DNA methylation at imprinting control regions in somatic cells.

-62 Baglivo I, Esposito S, De Cesare L et al. Genetic and epigenetic mutations affect the
DNA binding capability of human ZFP57 in transient neonatal diabetes type 1. FEBS Lett. 587(10), 1474-1481 (2013).

63 Sharif J, Muto M, Takebayashi S et al. The SRA protein Np95 mediates epigenetic inheritance by recruiting Dnmt1 to methylated DNA. Nature 450(7171), 908-912 (2007).

-64 Messerschmidt DM, de Vries W, Ito M, Solter D, Ferguson-Smith A, Knowles BB. Trim 28 is required for epigenetic stability during mouse oocyte to embryo transition. Science 335(6075), 1499-1502 (2012).

-65 Riclet R, Chendeb M, Vonesch JL et al. Disruption of the interaction between transcriptional intermediary factor $1 \beta$ and heterochromatin protein 1 leads to a switch from DNA hyper- to hypomethylation and $\mathrm{H} 3 \mathrm{~K} 9$ to $\mathrm{H} 3 \mathrm{~K} 27$ trimethylation on the MEST promoter correlating with gene reactivation. Mol. Biol. Cell 20(1), 296-305 (2009).

66 Kim JD, Kim H, Ekram MB, Yu S, Faulk C, Kim J. Rex1/Zfp42 as an epigenetic regulator for genomic imprinting. Hum. Mol. Genet. 20(7), 1353-1362 (2011).

-67 Meyer E, Lim D, Pasha S et al. Germline mutation in NLRP2 (NALP2) in a familial imprinting disorder (Beckwith-Wiedemann syndrome). PLoS Genet. 5(3), e1000423 (2009).

- This study on Beckwith-Wiedemann syndrome describes a familial, genetic mutation at the NLRP2 gene, which causes hypomethylation at the imprinting control region of the KCNQ1 domain.

-68 Kajii T, Ohama K. Androgenetic origin of hydatidiform mole. Nature 268(5621), 633-634 (1977).

69 Devriendt K. Hydatidiform mole and triploidy: the role of genomic imprinting in placental development. Hum. Reprod. Update 11(2), 137-142 (2005).

70 Van den Veyver IB, Al-Hussaini TK. Biparental hydatidiform moles: a maternal effect mutation affecting imprinting in the offspring. Hum. Reprod. Update 12(3), 233-242 (2006).

71 El-Maarri O, Seoud M, Coullin P et al. Maternal alleles acquiring paternal methylation patterns in biparental complete hydatidiform moles. Hum. Mol. Genet. 12(12), 1405-1413 (2003).

-72 Hayward BE, De Vos M, Talati N et al. Genetic and epigenetic analysis of recurrent hydatidiform mole. Hum. Mutat. 30(5), E629-E639 (2009).

Judson H, Hayward BE, Sheridan E, Bonthron DT. A global disorder of imprinting in the human female germ line. Nature 416(6880), 539-542 (2002).
74 Murdoch S, Djuric U, Mazhar B et al. Mutations in NALP7 cause recurrent hydatidiform moles and reproductive wastage in humans. Nat. Genet. 38(3), 300-302 (2006).

75 Wang CM, Dixon PH, Decordova S et al. Identification of 13 novel NLRP7 mutations in 20 families with recurrent hydatidiform mole; missense mutations cluster in the leucine-rich region. J. Med. Genet. 46(8), 569-575 (2009).

76 Parry DA, Logan CV, Hayward BE et al. Mutations causing familial biparental hydatidiform mole implicate c6orf221 as a possible regulator of genomic imprinting in the human oocyte. Am. J. Hum. Genet. 89(3), 451-458 (2011).

-77 Pierre A, Gautier M, Callebaut I et al. Atypical structure and phylogenomic evolution of the new eutherian oocyte- and embryo-expressed KHDC1/DPPA5/ECAT1/OOEP gene family. Genomics 90(5), 583-594 (2007).

78 Berland S, Appelback M, Bruland O et al. Evidence for anticipation in Beckwith-Wiedemann syndrome. Eur. J. Hum. Genet. (2013) (Epub ahead of print).

79 Demars J, Shmela ME, Rossignol S et al. Analysis of the IGF2/H19 imprinting control region uncovers new genetic defects, including mutations of OCT-binding sequences, in patients with 11p15 fetal growth disorders. Hum. Mol. Genet. 19(5), 803-814 (2010).

- This study on Beckwith-Wiedemann syndrome shows that OCT4-binding sites are required to prevent acquisition of aberrant DNA methylation at the imprinting control region of the IGF2/H19 domain.

80 Poole RL, Leith DJ, Docherty LE et al. Beckwith-Wiedemann syndrome caused by maternally inherited mutation of an OCTbinding motif in the IGF2/H19-imprinting control region, ICR1. Eur. J. Hum. Genet. 20(2), 240-243 (2012).

-81 Kaufman Y, Heled M, Perk J, Razin A, Shemer R. Protein-binding elements establish in the oocyte the primary imprint of the PraderWilli/Angelman syndromes domain. Proc. Natl Acad. Sci. USA 106(25), 10242-10247 (2009).

82 Hori N, Yamane M, Kouno K, Sato K. Induction of DNA demethylation depending on two sets of SOX2 and adjacent OCT3/4 binding sites (SOX-OCT motifs) within the mouse $H 19 /$ insulin-like growth factor 2 (IGF2) imprinted control region. J. Biol. Chem. 287(52), 44006-44016 (2012).

83 Sakaguchi R, Okamura E, Matsuzaki H, Fukamizu A, Tanimoto K. SOX-OCT motifs contribute to maintenance of the unmethylated H19 ICR in YAC transgenic mice. Hum. Mol. Genet. doi:10.1093/hmg/ddt311 (2013) (Epub ahead of print). 
84 Donohoe ME, Silva SS, Pinter SF, Xu N, Lee JT. The pluripotency factor Oct 4 interacts with Ctcf and also controls $\mathrm{X}$-chromosome pairing and counting. Nature 460(7251), 128-132 (2009).

85 Kim YJ, Cecchini KR, Kim TH. Conserved, developmentally regulated mechanism couples chromosomal looping and heterochromatin barrier activity at the homeobox gene A locus. Proc. Natl Acad. Sci. USA 108(18), 7391-7396 (2011).

86 Bell AC, West AG, Felsenfeld G. The protein CTCF is required for the enhancer blocking activity of vertebrate insulators. Cell 98(3), 387-396 (1999).

87 Giles KE, Gowher H, Ghirlando R, Jin C, Felsenfeld G. Chromatin boundaries, insulators, and long-range interactions in the nucleus. Cold Spring Harb. Symp. Quant. Biol. 75, 79-85 (2010).

88 Phillips JE, Corces VG. CTCF: master weaver of the genome. Cell 137(7), 1194-1211 (2009).

89 Bell AC, Felsenfeld G. Methylation of a CTCF-dependent boundary controls imprinted expression of the $I g f 2$ gene. Nature 405(6785), 482-485 (2000).

90 Hark AT, Schoenherr CJ, Katz DJ, Ingram RS, Levorse JM, Tilghman SM. CTCF mediates methylation-sensitive enhancer-blocking activity at the H19/IGF2 locus. Nature 405(6785), 486-489 (2000).

91 Kanduri C, Pant V, Loukinov D et al. Functional association of CTCF with the insulator upstream of the $\mathrm{H} 19$ gene is parent of origin-specific and methylation-sensitive. Curr. Biol. 10(14), 853-856 (2000).

-92 Murrell A, Heeson S, Reik W. Interaction between differentially methylated regions partitions the imprinted genes IGF2 and H19 into parent-specific chromatin loops. Nat. Genet. 36(8), 889-893 (2004).

93 Wendt KS, Yoshida K, Itoh T et al. Cohesin mediates transcriptional insulation by
CCCTC-binding factor. Nature 451(7180), 796-801 (2008).

94 Nativio R, Wendt KS, Ito Y et al. Cohesin is required for higher-order chromatin conformation at the imprinted IGF2-H19 locus. PLoS Genet. 5(11), e1000739 (2009).

-95 Engel N, Thorvaldsen JL, Bartolomei MS. CTCF binding sites promote transcription initiation and prevent DNA methylation on the maternal allele at the imprinted H19/IGF2 locus. Hum. Mol. Genet. 15(19), 2945-2954 (2006).

6 Schoenherr CJ, Levorse JM, Tilghman SM. CTCF maintains differential methylation at the IGF2/H19 locus. Nat. Genet. 33(1), 66-69 (2003).

97 Beygo J, Citro V, Sparago A et al. The molecular function and clinical phenotype of partial deletions of the IGF2/H19 imprinting control region depends on the spatial arrangement of the remaining CTCF-binding sites. Hum. Mol. Genet. 22(3), 544-557 (2013).

98 Choufani S, Shuman C, Weksberg R. Beckwith-Wiedemann syndrome. Am. J. Med. Genet. C Semin. Med. Genet. 154C(3), 343-354 (2010).

-99 Nakamura T, Arai Y, Umehara H et al. PGC7/ Stella protects against DNA demethylation in early embryogenesis. Nat. Cell Biol. 9(1), 64-71 (2007).

"- This study shows that the PGC7/Stella protein is essential for the early embryonic maintenance of DNA methylation at several imprinting control regions.

100 Olek A, Walter J. The pre-implantation ontogeny of the $H 19$ methylation imprint. Nat. Genet. 17(3), 275-276 (1997).

101 Liu YJ, Nakamura T, Nakano T. Essential role of DPPA3 for chromatin condensation in mouse oocytogenesis. Biol. Reprod. 86(2), 40 (2012).

102 Nakamura T, Liu YJ, Nakashima H et al. PGC7 binds histone $\mathrm{H} 3 \mathrm{~K} 9 \mathrm{me} 2$ to protect against conversion of $5 \mathrm{mC}$ to $5 \mathrm{hmC}$ in early embryos. Nature 486(7403), 415-419 (2012).

103 Wossidlo M, Nakamura T, Lepikhov K et al. 5-Hydroxymethylcytosine in the mammalian zygote is linked with epigenetic reprogramming. Nat. Commun. 2, 241 (2011).

104 Wu MY, Tsai TF, Beaudet AL. Deficiency of Rbbp1/Arid4a and Rbbp1l1/Arid4b alters epigenetic modifications and suppresses an imprinting defect in the PWS/AS domain. Genes Dev. 20(20), 2859-2870 (2006).

105 Reese KJ, Lin S, Verona RI, Schultz RM, Bartolomei MS. Maintenance of paternal methylation and repression of the imprinted H19 gene requires MBD3. PLoS Genet. 3(8), e137 (2007).

- 106 Ma P, Lin S, Bartolomei MS, Schultz RM. Metastasis tumor antigen 2 (MTA2) is involved in proper imprinted expression of $H 19$ and Peg3 during mouse preimplantation development. Biol. Reprod. 83(6), 1027-1035 (2010).

107 Keren B, Chantot-Bastaraud S, Brioude F et al. SNP arrays in Beckwith-Wiedemann syndrome: an improved diagnostic strategy. Eur. J. Med. Genet. 56(10), 546-550 (2013).

108 Borgel J, Guibert S, Li Y et al. Targets and dynamics of promoter DNA methylation during early mouse development. Nat. Genet. 42(12), 1093-1100 (2010).

109 Kelsey G, Feil R. New insights into establishment and maintenance of DNA methylation imprints in mammals. Philos. Trans. R Soc. Lond. B Biol. Sci. 368(1609), 20110336 (2013).

\section{Website}

201 Williamson CM, Blake A, Thomas S et al. Mouse imprinting data and references (2013). www.har.mrc.ac.uk/research/genomic imprinting 